\title{
Charakteristika von Palliativpatienten mit Atemnot
}

\section{Ergebnisse der deutschlandweiten Hospiz- und Palliativerhebung}

\author{
S. T. Simon ${ }^{1,2,3,4 *}$, N. Altfelder ${ }^{1 *}$, B. Alt-Epping ${ }^{5}$, C. Bausewein ${ }^{1,6}$, V. Weingärtner ${ }^{1,2}$, R. Voltz ${ }^{2,3,4}$, C. Ostgathe ${ }^{7}$, \\ L. Radbruch ${ }^{8,9}$, G. Lindena ${ }^{10}$, F. Nauck ${ }^{5}$
}

\section{Zusammenfassung}

Hintergrund I Atemnot ist ein häufiges und belastendes Symptom bei Patienten mit fortgeschrittener lebenslimitierender Erkrankung. Das Ziel dieser Untersuchung war die Beschreibung demografischer und klinischer Charakteristika von Patienten mit Atemnot in Deutschland.

Methoden | Wir führten eine Sekundäranalyse von Patientendaten der nationalen Erhebung aus spezialisierten stationären Hospiz- und Palliativeinrichtungen von 2006 bis 2008 durch. Die Hospiz- und Palliativ-Erfassung (HOPE) erhebt jährlich prospektiv deutschlandweit u.a. anhand einer validierten Checkliste standardisiert 16 Symptome und Probleme (Schweregrad 0-3).

Die Charakteristika von Patienten mit Atemnot $(\mathrm{PmA})$ und ohne (PoA) wurden rein deskriptiv verglichen. Bei der Interpretation der angegebenen $\mathrm{p}$-Werte wurde die Fehlerinflation aufgrund multipler Tests berücksichtigt.

\section{Hintergrund}

Atemnot ist ein häufiges und belastendes Symptom bei Patienten mit einer fortgeschrittenen, unheilbaren und lebensbedrohlichen Erkrankung. Atemnot wird von der American Thoracic Society definiert als “...ein subjektives Erleben erschwerter Atmung, das in seiner Ausprägung schwanken kann. Die Art der Empfindung wird von einem komplexen Zusammenspiel physiologischer, psychologischer, sozialer und umweltbedingter Faktoren beeinflusst“" [23].

Die Prävalenz von Atemnot unterscheidet sich je nach der Grunderkrankung und nimmt mit nahendem Tod zu [10, 20]. Die Prävalenz von Atemnot bei Tumorerkrankungen variiert zwischen 16-77\%, abhängig von der Tumorentität und der Art der Erhebung [10, 20, 29]. Atemnot ist am häufigsten bei Patienten

- mit Lungenkrebs (72\%) [3],

- chronischer Herzinsuffizienz (88\%) [20],

- interstitieller Lungenerkrankung (93\%) [5] und

- bei einer chronisch obstruktiven Lungenerkrankung (COPD) (bis zu 98\%) [20].
Ergebnisse I Bei 2860/5320 (53,8\%) Patienten wurde Atemnot dokumentiert (Durchschnittsalter 67,2 $\pm 12,4$ Jahre, 51,4\% der Patienten waren weiblich. 2676 Patienten mit Atemnot (93,6\%) hatten eine maligne Grunderkrankung (weiblich / männlich:

- Lungenkrebs: $15,7 \% / 29,5 \%$

- Brustkrebs: 20,3\%/0,3\%

- Dickdarmkrebs: $10,3 \% / 8,7 \%$

PmA hatten einen signifikant schlechteren Funktionsstatus als PoA (ECOG 3/4: 78,4\% vs. 70,8\%, $\mathrm{p}<0,001$ ) und eine höhere Symptomanzahl und -Intensitätslast (außer Verwirrtheit). PmA hatten ein höheres assoziiertes Risiko, während des stationären Aufenthalts zu sterben (43,5\% vs. $32,4 \%$, $\mathrm{p}<0,001)$.

Folgerung I In dieser umfassenden Studienpopulation aus spezialisierten stationären Hospiz- und Palliativeinrichtungen war Atemnot mit einer höheren Symptomanzahl und einer geringeren Überlebenszeit assoziiert. PmA benötigen eine besondere Aufmerksamkeit in der Versorgung.

In Deutschland leben 80,5 Mio. Menschen, davon sind 46,7 Mio. über 40 Jahre alt [31]. Basierend auf aktuellen Prävalenzangaben für die fortgeschrittene COPD (0,8\% im Alter $\geq 40$ Jahre, GOLD Stadien III-IV [13]) oder den fortgeschrittenen Lungenkrebs (76100, 5-Jahresprävalenz [27], $32 \% / 36 \%$ Stadium III/IV [7]) leiden etwa 37200 Patienten mit Lungenkrebs und 365500 Patienten mit COPD unter Atemnot.

Atemnot schränkt sowohl die Lebensqualität der Patienten als auch ihrer Angehörigen stark ein $[14,15]$. Obwohl das Symptom Atemnot und seine Behandlungsoptionen zunehmend wissenschaftlich erforscht werden, gibt es bisher wenige Untersuchungen über die demografischen und klinischen Charakteristika von Patienten. Es ist außerdem bisher nicht eindeutig geklärt, ob das Vorhandensein und die Intensität von Atemnot zur Abschätzung der Überlebenszeit von schwer kranken Patienten herangezogen werden kann [32].

Deshalb war das Ziel der vorliegenden Arbeit, die demografischen und klinischen Charakteristika von Patienten mit Atemnot (PmA) im Vergleich
Institute

1 Institut für Palliative Care, Oldenburg

2 Zentrum für Palliativmedizin, Uniklinikum Köln

3 Klinisches Studienzentrum Palliativmedizin, Uniklinikum Köln

4 Centrum für Integrierte Onkologie Köln Bonn, Uniklinikum Köln

5 Klinik für Palliativmedizin, Universitätsmedizin Göttingen

6 Klinik und Poliklinik für Palliativmedizin, Klinikum der Universität München

7 Palliativmedizinische Abteilung, Universitätsklinikum Erlangen, CCC EMN, Erlangen

8 Klinik für Palliativmedizin, Universitätsklinikum Bonn

9 Zentrum für Palliativmedizin, Malteser Krankenhaus Bonn / Rhein-Sieg, Bonn

10 CLARA Klinische Forschung, Kleinmachnow

* geteilte Erstautorenschaft

\section{Korrespondenz}

PD Dr. med.

Steffen Simon MSc

Zentrum für Palliativmedizin, Uniklinik Köln

Kerpener Str. 62 50937 Köln

Tel 0049-221-47897645 Fax 0049-221-47889300 steffen@steffensimon.de 
zu Patienten ohne Atemnot (PoA) aus spezialisierten stationären Hospiz- und Palliativeinrichtungen in Deutschland zu beschreiben.

\section{Methoden}

Die vorliegende Untersuchung ist eine Sekundäranalyse von prospektiv multizentrisch erhobenen nationalen Patientendaten (Beobachtungsstudie) aus stationären spezialisierten Hospizund Palliativeinrichtungen in Deutschland. Die erhobenen Daten wurden im Querschnitt (Zeitpunkt der stationären Aufnahme) sowie im Längsschnitt (Überleben) ausgewertet. Die Studie wurde von der Ethikkommission der Universität Göttingen genehmigt (Antragsnummer 3/2/09).

Datensatz I Die Hospiz- und Palliativ-Erfassung (HOPE, www.hope-clara.de) ist eine jährliche, prospektive, standardisierte, deutschlandweite Erhebung, in der seit 1999 demografische und klinische Patientendaten gesammelt werden. An HOPE nehmen verschiedene Einrichtungen teil:
- Palliativstationen

- Hospizen

- onkologische Abteilungen

- palliativmedizinische Dienste

- ambulante palliativärztlichen und -pflegerische Diensten

Über einen Zeitraum von drei Monaten werden in den teilnehmenden Einrichtungen konsekutiv 30 Patienten bei der Aufnahme und Entlassung bzw. beim Todeszeitpunkt anonymisiert erfasst. Der sogenannte Basisbogen bildet den Kerndatensatz und umfasst unter anderem:

- demografische Patientendaten

- klinische Angaben

- Diagnose

$\checkmark$ Krankheitsstadium

- Symptom- und Problemcheckliste

$\checkmark$ Therapie

$\checkmark$ Medikation

Daten aus HOPE wurden bereits mehrfach für wissenschaftliche Studien herangezogen $[2,21$, 24, 29, 33].
Tab. 1 Patientencharakteristika.

\begin{tabular}{|c|c|c|c|c|}
\hline & $\begin{array}{l}\text { Gesamt } \\
n=5320(\%)\end{array}$ & $\begin{array}{l}\mathrm{PmA} \\
\mathrm{n}=2860(\%)\end{array}$ & $\begin{array}{l}\text { PoA } \\
n=2460(\%)\end{array}$ & p-Wert ${ }^{a}$ \\
\hline Alter, MW (SD) & $67,2(13,0)$ & $67,2(12,4)$ & $67,2(13,5)$ & 0,960 \\
\hline Geschlecht weiblich ${ }^{\mathrm{b}}$ & $2839(53,4)$ & $1469(51,4)$ & $1370(55,7)$ & 0,001 \\
\hline Maligne Diagnose $^{c}$ & $5014(94,2)$ & $2676(93,6)$ & $2338(95,0)$ & $0,030^{\mathrm{e}}$ \\
\hline Bronchialkarzinom & $846(15,9)$ & $642(22,4)$ & $222(9,0)$ & \\
\hline Kolorektales Karzinom & $575(10,8)$ & $271(9,5)$ & $304(12,4)$ & \\
\hline Mammakarzinom & $549(10,3)$ & $304(10,6)$ & $245(10,0)$ & \\
\hline Pankreaskarzinom & $322(6,0)$ & $145(5,1)$ & $177(7,2)$ & \\
\hline Prostatakarzinom & $235(4,4)$ & $101(3,5)$ & $134(5,4)$ & \\
\hline Metastasen & $4323(81,3)$ & $2378(88,9)$ & $1945(83,2)$ & $<0,001$ \\
\hline Nicht-maligne Diagnose & $289(5,4)$ & $173(6,1)$ & $116(4,7)$ & 0,030 \\
\hline COPD & $22(0,4)$ & $20(0,7)$ & $2(0,1)$ & 0,002 \\
\hline $\mathrm{CHF}$ & $14(0,2)$ & $18(0,6)$ & $6(0,2)$ & 0,110 \\
\hline ECOG $3-4^{d}$ & $3986(74,9)$ & $2244(78,4)$ & $1742(70,8)$ & $<0,001$ \\
\hline Hilfe bei ADL benötigt & $4847(91,1)$ & $2657(92,9)$ & $2190(89,0)$ & $<0,001$ \\
\hline Symptomanzahl, MW (SD) & & $8,5(2,1)$ & $7,0(2,2)$ & $<0,001$ \\
\hline Symptomintensitätslast, MW (SD) & & $17,1(5,7)$ & $13,7(5,4)$ & $<0,001$ \\
\hline \multicolumn{5}{|c|}{$\begin{array}{l}\text { Die Prozent-Angaben beziehen sich je auf die entsprechende Gesamtzahl an Patienten in der Gruppe } \\
\text { (gesamt, PmA, PoA). } \\
\text { a Vergleich der Gruppe PmA und PoA, p-Wert ist fett markiert, wenn statistisch signifikant }(p<0,05) \\
{ }^{b} \text { Fehlende Daten: gesamt n }=61(1,1 \%), \operatorname{PmA} 32(1,1 \%), \operatorname{PoA} 29(1,2 \%) \\
{ }^{c} \text { Fehlende Daten: gesamt n=17 (0,3\%), PmA } 11(0,4 \%), \operatorname{PoA} 6(0,2 \%) \\
{ }^{d} \text { Fehlende Daten: gesamt n=334 (6,3\%), PmA } 178(6,2 \%), \text { PoA } 156(6,3 \%) \\
\text { e Test bezieht sich auf alle Patienten mit einer malignen Grunderkrankung } \\
\text { ADL: Aktivitäten des täglichen Lebens, ECOG: Eastern Cooperative Oncology Group, MW: Mittelwert; } \\
\text { PmA: Patienten mit Atemnot, PoA: Patienten ohne Atemnot, SD: Standardabweichung }\end{array}$} \\
\hline
\end{tabular}


Die vorliegende Untersuchung beruht auf HOPEDaten aus spezialisierten stationären Hospiz- und Palliativeinrichtungen aus den Jahren 20062008. Für die Ermittlung der Sterbefälle und der Liegezeiten wurden als zweiter Dokumentationszeitpunkt die Entlassung, der Tod oder das Ende der Dokumentationsphase herangezogen.

Patienten und Umfeld | Patienten, die den folgenden Kriterien entsprachen, wurden in die Analyse eingeschlossen:

1. Die Aufnahme der Patienten erfolgte in eine spezialisierte stationäre Hospiz- oder Palliativeinrichtung, da Aufnahmekriterien und Umfeld in diesen Institutionen eine gute Vergleichbarkeit der Daten zuließen (relativ homogene Stichprobe).

2. Die Daten aus den Jahren 2006 bis 2008 wurden gewählt, da die Dokumentation aus diesem Zeitraum weitestgehend identisch aufgebaut war und so eine valide Datensynthese ermöglichte.

3. Auf dem Dokumentationsbogen lag eine Aussage zum Vorhandensein oder Fehlen des Symptoms Atemnot vor.

Instrumente | Im HOPE Basisbogen wurden mittels Fremdeinschätzung durch ein professionelles Mitglied des Behandlungsteams folgende Angaben dokumentiert:

- Alter

- Geschlecht

- Institution

- Funktionsstatus

- Diagnose

- Krankheitsstadium

- ggfs. Tod während des stationären Aufenthaltes

- Vorhandensein und Schweregrad von Symptomen

Der Funktionsstatus wurde nach der Klassifikation der Eastern Cooperative Oncology Group (ECOG, 0-4) eingestuft. Die validierte Symptomund Problemliste umfasst folgende 16 Symptome (12) und Probleme (4), die auf einer vierstufigen Likert-Skala $(0=$ kein, $1=$ leicht, $2=$ mittel, $3=$ stark) angegeben wurden [33]:

- Schmerzen

- Übelkeit

- Erbrechen

- Luftnot (im Folgenden „Atemnot“)

- Verstopfung

- Schwäche

- Appetitmangel

- Müdigkeit

- Depressivität

- Angst

- Anspannung

- Desorientiertheit/Verwirrtheit

- Pflegeprobleme wegen Wunden/Dekubitus

- Hilfebedarf bei Aktivitäten des täglichen Lebens

- Probleme mit der Organisation der Versorgung

- Überforderung der Familie/des Umfeldes

\section{Prävalenz von Atemnot in \%}

\begin{tabular}{ll}
\hline Krebsdiagnose $^{\mathbf{a}}$ & \\
\hline Bronchialkarzinom & 74,3 \\
Mammakarzinom & 55,4 \\
\hline Karzinom des Mund- und Rachenraums & 55,3 \\
\hline Ovarialkarzinom & 53,6 \\
\hline Karzinom des Leber-Gallenwegssystems & 47,4 \\
\hline kolorektales Karzinom & 47,1 \\
\hline Nierenkarzinom & 46,9 \\
\hline Pankreaskarzinom & 45,0 \\
\hline Prostatakarzinom & 43,0 \\
\hline Magenkarzinom & 42,0 \\
\hline Funktionsstatus (ECOG) & \\
\hline 0 & 25,0 \\
1 & 38,4 \\
\hline 2 & 45,7 \\
\hline 3 & 55,3 \\
4 & 57,1
\end{tabular}

${ }^{a}$ Gezeigt sind die zehn häufigsten Tumorentitäten basierend auf den PmA.

${ }^{b}$ Fehlende Daten: 178 (6,3\%)

ECOG: Eastern Cooperative Oncology Group

Tab. 2 Atemnotprävalenz nach Tumorentität ${ }^{\mathrm{a}}$ und Funktionsstatus der PmA

Analyse | Es wurde eine rein deskriptive Analyse durchgeführt. Die Beschreibung der Studienpopulation und der Charakteristika erfolgte mittels absoluten und relativen Häufigkeiten (bei Normalverteilung mittels Mittelwert [MW] und Standardabweichung [SD]; bei nicht normalverteilten Daten mittels Median und Spannweite [SpW]).

Für jeden Patienten wurde die Symptomanzahl als Summe der vorhandenen Symptome berechnet $(0-12)$ sowie die Symptomintensitätslast $(0-$ 36) mittels Aufsummieren der Schweregrade ( 0 3) der vorhandenen Symptome bestimmt. Für die Analyse wurden PmA und PoA unterschieden und verglichen.

Zum Vergleich nominal-skalierter Daten wurde der Chi-Quadrat-Test, für ordinal skalierte Daten der Mann-Whitney-U-Test und für kontinuierliche Daten der t-Test für unverbundene Stichproben angewendet. Das Signifikanzniveau wurde durchweg (und unkorrigiert) auf $\alpha=0,05$ festgesetzt. Bei Vorhandensein multipler Testungen wurden signifikante Ergebnisse mit angemessener Vorsicht interpretiert.

Die (mediane) Überlebenszeit wurde für die Zeit des stationären Aufenthaltes bestimmt, für PmA und PoA anhand einer Kaplan-Meier-Kurve beschrieben und mittels des Log-Rang-Tests vergli- 
Abb. 1 Kaplan-Meier-Kurven zur Überlebenszeit während des stationären Aufenthaltes aller verstorbenen Patienten (zensierte Beobachtungen) getrennt nach der Atemnotintensität (keine-leichtmittel-stark).

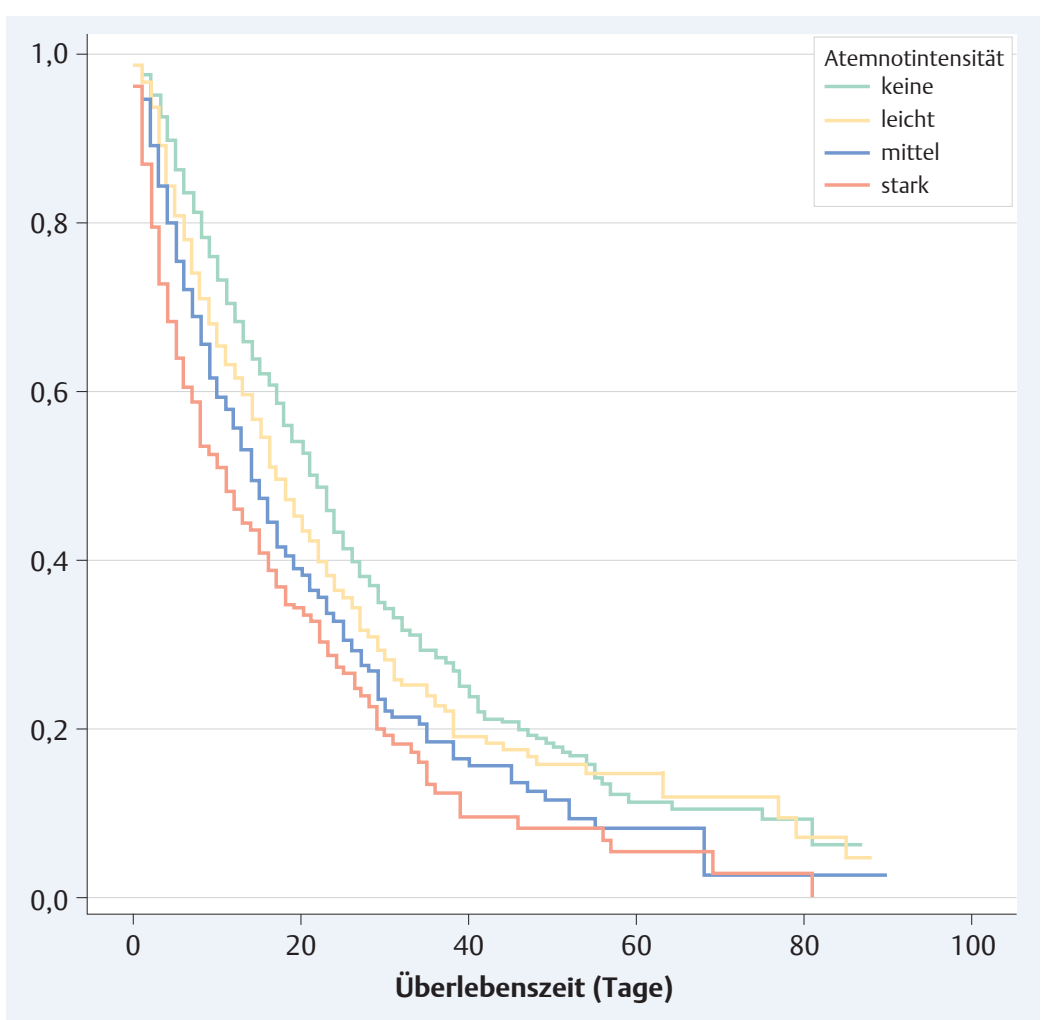
zentwert) bestimmt („Modell-Nutzen“ angenommen bei $\geq 20 \%$ ) [22].

Die Analysen wurden mittels der Statistiksoftware SPSS (Version 17.0) durchgeführt.

\section{Ergebnisse}

chen. Wenn der Patient lebend entlassen oder das Ende der Dokumentationsphase erreicht wurde, wunden die Daten zensiert.

Potenzielle Risikofaktoren für Atemnot wurden auf Basis einer Korrelationsanalyse und der klinischen Relevanz ausgewählt, weiterhin mittels logistischer Regression (univariable Analyse) untersucht und durch das Chancenverhältnis (Odds Ratio (OR) beschrieben. Um die Stärke des Zusammenhangs zwischen Atemnot und Patientenmerkmalen / Symptomen einheitlich zu beschreiben, wurde stets Spearman's rho berechnet. Dieser Koeffizient stellt sehr geringe Anforderungen an das Skalenniveau und ist sogar für binäre Merkmale („Phi-Koeffizient“) interpretierbar (Ergebnisse sind hier nicht gezeigt). Die Korrelationsergebnisse wurden nach Cohen gewertet [8]:

- 0,29-0,10 geringer Zusammenhang

- 0,30-0,49 mäßiger Zusammenhang

- 0,50-1,00 großer Zusammenhang

Auf der Basis der o.g. univariablen Analyse (Risikofaktoren mit der höchsten OR) wurden die potenziellen Risikofaktoren für Atemnot einer multivariablen Regressionsanalyse zugeführt mit dem Ziel einer Modellentwicklung. Die durch das Modell erklärte Varianz wurde mittels Cox-\&-Snell-R-Quadrat-Test (erster Prozentwert) und Nagelkerke-R-Quadrat-Test (zweiter Pro-

Datensatz | Die Daten von 5320 Patienten (2006: 1861, 2007: 2066, 2008: 1393) wurden in die Analyse eingeschlossen ( Tab. 1). Das Durchschnittsalter der Patienten betrug 67,2 $\pm 13,0$ Jahre (MW $\pm S D$ ), 53,4\% waren weiblich, 79,5\% wurden auf einer Palliativstation versorgt und 20,5\% in einem stationären Hospiz. Die Mehrheit der Patienten litt an einer malignen Grunderkrankung $(94,3 \%)$ und hatte einen schlechten Funktionsstatus (74,9\% mit ECOG 3 oder 4, Spannweite 0-4). 38,4\% aller Patienten starben während des stationären Aufenthaltes, 37,0\% überlebten (24,7\% fehlende Daten). Die dokumentierte Prävalenz von Atemnot betrug insgesamt 53,8\% $(\mathrm{n}=2860$ Patienten, $\triangleright$ Tab. $\mathbf{1})$.

Vergleich der Patienten mit und ohne Atemnot I Für PmA ergab sich im Vergleich zu PoA ( Tab. 1) ein signifikant

- höherer Anteil an männlichen Patienten ( $47,5 \%$ vs. $43,1 \%, p=0,001$ ),

- der Diagnose Lungenkrebs,

- einem eingeschränkten Funktionsstatus sowie

- einem höheren Hilfebedarf bei Aktivitäten des täglichen Lebens.

Bei PmA waren die Symptomanzahl $(8,5 \pm 2,1$ vs $7,0 \pm 2,2, \mathrm{p}<0,001)$ und -intensitätslast $(17,1 \pm 5,7$ vs. $13,7 \pm 5,4, p<0,001)$ signifikant erhöht. Für alle Symptome bis auf Erbrechen und Desorientiertheit ergab sich eine signifikant höhere Prävalenz bei PmA im Vergleich zu PoA.

In der Gruppe der Krebspatienten hatten PmA signifikant häufiger Metastasen als PoA ( $\bullet$ Tab. 1). PmA hatten am häufigsten

- Lungen- (42,5\%),

- Leber- $(35,3 \%)$ und

- Knochenmetastasen (32,2\%);

PoA im Vergleich

- Leber- (33,5\%),

- Knochen- $(30,9 \%)$ und

- Lungenmetastasen $(23,1 \%)$.

Unter den Patienten mit nicht-maligner Erkrankung hatten signifikant mehr PmA eine COPD $(11,6 \%$ vs. $1,7 \%, p=0,002)$.

Symptomausprägung bei Patienten mit Atemnot | Die Atemnotintensität wurde von 56,6\% der Patienten als mittel oder stark angegeben (Median 2; SpW 1-3). Unter den 2676 PmA mit einer Krebsdiagnose unterschied sich die Atemnotprävalenz zwischen den Tumorentitäten. \ Tab. 2 zeigt die Prävalenz für die zehn häufigsten Tumorentitäten der PmA.

Überlebenszeit | Während des stationären Aufenthaltes starben insgesamt 2024 Patienten $(38,4 \%)$ und 1966 (37,0\%) überlebten $(24,7 \%$ fehlende Daten). Es starben signifikant mehr PmA als PoA (43,5\% vs. 32,4\%, p<0,001). Die Überlebenszeit der, Patienten, die während des stationären 


\begin{tabular}{|c|c|c|c|c|}
\hline Variable & n & OR & $95 \%-K I$ & $\mathbf{p}$ \\
\hline weiblich & 2839 & 1 & & \\
\hline männlich & 2420 & 1,2 & $1,07-1,33$ & 0,001 \\
\hline Altersgruppen & & & & 0,179 \\
\hline$<65$ & 1910 & 1,22 & $0,98-1,52$ & 0,072 \\
\hline $65-74$ & 1679 & 1,21 & $0,97-1,51$ & 0,093 \\
\hline $75-84$ & 1233 & 1,1 & $0,88-1,39$ & 0,41 \\
\hline$>84$ & 382 & 1 & & \\
\hline Allein lebend & 814 & 1 & & \\
\hline nicht allein lebend & 2428 & 1,08 & $0,92-1,27$ & 0,34 \\
\hline Krebs & 5014 & 1 & & \\
\hline kein Krebs & 289 & 1,3 & $1,02-1,66$ & 0,03 \\
\hline Bösartige Neubildung in der Lunge & 2169 & 2,71 & $2,42-3,04$ & $<0,001$ \\
\hline keine Neubildung in der Lunge & 3151 & 1 & & \\
\hline ECOG & & & & $<0,001$ \\
\hline 0 & 20 & 1 & & \\
\hline 1 & 198 & 1,87 & $0,65-5,35$ & 0,244 \\
\hline 2 & 782 & 2,52 & $0,91-7,00$ & 0,076 \\
\hline 3 & 1759 & 3,71 & $1,34-10,26$ & 0,011 \\
\hline 4 & 2227 & 3,99 & $1,45-11,01$ & 0,008 \\
\hline Schwäche ja & 5166 & 2,69 & $1,73-4,18$ & $<0,001$ \\
\hline nein & 95 & 1 & & \\
\hline 0 & 95 & 1 & & \\
\hline 1 & 496 & 1,31 & $0,82-2,10$ & 0,27 \\
\hline 2 & 1694 & 2,17 & $1,39-3,39$ & 0,001 \\
\hline 3 & 2976 & 3,44 & $2,21-5,35$ & $<0,001$ \\
\hline Depressivität ja & 3187 & 1,39 & $1,24-1,56$ & $<0,001$ \\
\hline nein & 1784 & 1 & & \\
\hline 0 & 1784 & 1 & & \\
\hline 1 & 1537 & 1,33 & $1,16-1,53$ & $<0,001$ \\
\hline 2 & 1080 & 1,32 & $1,14-1,54$ & $<0,001$ \\
\hline 3 & 570 & 1,72 & $1,42-2,09$ & $<0,001$ \\
\hline Angst ja & 3554 & 1,69 & $1,49-1,91$ & $<0,001$ \\
\hline nein & 1475 & 1 & & \\
\hline 0 & 1475 & 1 & & \\
\hline 1 & 1643 & 1,3 & $1,123-1,50$ & $<0,001$ \\
\hline 2 & 1238 & 1,94 & $1,67-2,26$ & $<0,001$ \\
\hline 3 & 673 & 2,56 & $2,11-3,10$ & $<0,001$ \\
\hline
\end{tabular}

ECOG: Eastern Cooperative Oncology Group, KI: Konfidenzintervall, OR: Odds ratio. $\mathrm{p}$-Wert ist fett markiert, wenn statistisch signifikant $(\mathrm{p}<0,05)$

Tab. 3 Ergebnisse der univariablen Analyse. 
Aufenthaltes gestorben sind, war bei PmA signifikant kürzer als bei PoA. Die mediane Überlebenszeit der PmA hing auch von der Schwere der Atemnotintensität ab $(\checkmark$ Abb. 1, Log-Rang-Test $\mathrm{p}<0,001)$ :

- PoA: 23 Tage

- PmA leichte Atemnot: 19 Tage

- PmA mittlere Atemnot: 16 Tage

- PmA starke Atemnot: 12 Tage, Log-Rang-Test $\mathrm{p}<0,001)$.

Faktoren, die mit Atemnot assoziiert sind I Die Ergebnisse der univariablen Analyse sind in - Tab. 3 zusammengefasst.

Im Modell der multivariablen Regression wurden folgende Faktoren berücksichtigt:

- maligne Neubildung (Karzinom oder Metastasen) der Lunge

- Funktionsstatus

- Schwäche

Verglichen mit der univariablen Analyse zeigte sich im Modell ein noch höheres assoziiertes Risiko für Atemnot bei Patienten mit maligner Neubildung (Karzinom oder Metastasen) der Lunge (OR 2,72; 95\%-Konfidenzintervall [2,40-3,07]; $\mathrm{p}<0,001)$. Weiterhin gab es einen signifikanten Zusammenhang zwischen Atemnot und

- Schwäche $(1,96 ;[1,19-3,22] ; \mathrm{p}=0,008)$,

- Angst (1,58; [1,39-1,81]); $\mathrm{p}<0,001)$ sowie

- insbesondere einem stark eingeschränkten Funktionsstatus (ECOG 4: 3,22; [1,11-9,27]; $\mathrm{p}=0,030)$.

Das Modell zeigt allerdings keinen Nutzen, da es lediglich 5,9-7,9\% der Varianz erklären kann.

\section{Diskussion}

Auf der Basis einer nationalen Datenanalyse konnte erstmalig die klinisch relevante Prävalenz und Intensität von Atemnot bei Patienten mit einer nicht heilbaren und fortgeschrittenen Grunderkrankung (vor allem Krebs) für Deutschland beschrieben werden. Hierbei zeigt sich das Vorliegen von Atemnot als Risikofaktor für eine höhere Symptomanzahl und -intensitätslast sowie für früheren Tod. Dies weist auf die Notwendigkeit einer palliativmedizinischen Behandlung und Begleitung dieser Patientengruppe hin.

Prävalenz | In Deutschland litten mit 53,8\% mehr als die Hälfte aller Patienten bei stationärer Aufnahme in eine spezialisierte Hospiz- oder Palliativeinrichtung unter dem Symptom Atemnot in einer meist mittelstarken Ausprägung. Dieses Ergebnis steht im Einklang mit den Daten großer internationaler Studien über Patienten mit fortgeschrittenen Krebserkrankungen:

- USA: Atemnotprävalenz von 49,3\% 5-6 Wochen vor dem Tod [26] und 50\% bei Patienten, die durchschnittlich noch zwei Monate lebten [35]
- Australien:50\% drei Monate vor Lebensende mit einem Anstieg auf 65\% in der letzten Lebenswoche [10].

In einer aktuellen multizentrischen Beobachtungsstudie über Patienten mit fortgeschrittenem Lungenkrebs in den USA $(n=450)$, Frankreich $(n=613)$ und Deutschland $(n=600)$ wurde jüngst eine besonders hohe Symptombelastung durch Atemnot (>90\%) sowie ihr negativer Effekt auf die Lebensqualität dieser Patientengruppe beschrieben $[18,19]$.

Symptome und Funktionsstatus | Die Analyse der Symptome demonstriert, dass PmA an einer bedeutsamen Anzahl weiterer belastender Symptome leiden. Bei PmA ergab sich sowohl bei der Anzahl der Symptome als auch bei der Symptomintensitätslast eine signifikant höhere mittlere Gesamtsumme und somit eine höhere Gesamtbelastung dieser Patientengruppe.

Fast alle untersuchten Symptome traten häufiger in der Atemnotgruppe auf. Eine besonders deutliche Differenz ergab sich bei den psychischen Symptomen

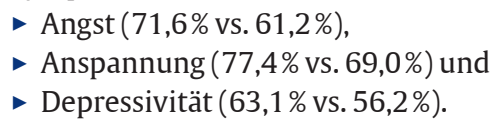

Hinweise auf eine besonders hohe Symptombelastung bei Patienten mit Atemnot [6] sowie statistisch signifikante Zusammenhänge zwischen Atemnot und den Symptomen Angst und Depressivität bzw. Depression bei Krebspatienten wurden für kleinere Stichproben bereits auch in anderen prospektiven Studien beschrieben [17, 25, 30]. In einer Kohorte von 624 Lungenkrebspatienten wurden bei PmA im Vergleich zu PoA statistisch signifikant häufiger Symptome einer Panikstörung berichtetet $(14,7 \%$ vs. $7,6 \%, p=0,004)$ [28].

PmA hatten im Vergleich zu PoA zusätzlich einen signifikant niedrigeren Funktionsstatus. Dies wurde bereits auch in vergleichbaren Patientengruppen beschrieben [16, 36]. Dieses Ergebnis unterstreicht, dass es sich bei PmA um eine Gruppe schwerkranker Patienten handelt, die besondere Aufmerksamkeit und Zuwendung benötigt.

Prognose I Die Ergebnisse der vorliegenden Arbeit unterstreichen, dass das Symptom Atemnot bei schwerkranken Patienten mit einer geringeren Überlebenszeit verbunden ist und als Risikofaktor für einen früheren Tod angesehen werden kann. Dabei scheint allein das Vorhandensein von Atemnot die Prognose zu verschlechtern. Noch deutlicher zeigte sich diese Tendenz jedoch bei der Betrachtung der Atemnotintensitäten: Mit zunehmender Atemnot sank die Überlebenszeit.

Eine systematische Übersichtsarbeit von Trajkovic-Vidakovic et al. stützt diese prognostische 
Funktion des Symptoms Atemnot bei Krebspatienten auf Basis von 35 Studien [34]. Der Zusammenhang war in $63 \%$ der identifizierten univariablen und 31\% der multivariablen Analysen statistisch signifikant. Cuervo Pinna et al. kamen bei 195 Krebspatienten im terminalen Stadium zu dem Schluss, dass PmA zwar ein signifikant erhöhtes Risiko hatten im Krankenhaus zu sterben (OR 2,13, p=0,010); jedoch fanden sie keinen signifikanten Zusammenhang zwischen Atemnot und der Überlebenszeit [9]. Darüber hinaus wurde ein Zusammenhang zwischen Atemnot und einem erhöhten Sterberisiko in 3 großen epidemiologischen Studien bei COPD-Patienten und der Allgemeinbevölkerung beschrieben $[1,11,12]$.

Limitationen | Die vorliegende Sekundäranalyse basiert auf nationalen Daten, bei denen methodische Einschränkungen bezüglich der Datenerhebung nicht ausgeschlossen werden können. Die jährliche, standardisierte und mit Hilfsmaterialen unterstützte Datenerhebung versucht jedoch eine bestmögliche Datenqualität zu erreichen. Den Limitationen einer Routineerhebung steht die umfassende Stichprobe von 5320 Patienten gegenüber. Mit dem Ziel, eine relativ homogene und gut vergleichbare Patientengruppe zu beschreiben, wurde der Fokus auf Patienten der stationären spezialisierten Hospiz- und Palliativversorgung gerichtet, was die Übertragbarkeit auf Patienten in der ambulanten Palliativversorgung einschränkt.

Die ermittelten mit dem Symptom Atemnot assoziierten Faktoren sind anhand dieser Analyse lediglich deskriptiv bzw. explorativ auf Basis von Querschnittsdaten beschrieben. Zur Klärung von Kausalzusammenhängen des zeitlichen Verlaufs bedarf es prospektiv vergleichender Studien. Ebenso wenig kann aus den Ergebnissen geschlossen werden, dass Atemnot zu Depressivität bzw. Depression und Angst führt.

Die Patienten stammten aus 26,1\% aller nationalen stationären Hospiz- und Palliativeinrichtungen und ermöglichen damit einen guten Überblick zur Versorgungslage in Deutschland. Da sämtliche Hospiz- und Palliativeinrichtungen in Deutschland zur Teilnahme an HOPE aufgerufen waren, kann bei dieser Rücklaufquote allerdings ein Selektionsbias nicht ausgeschlossen werden. Die Daten beruhen auf einer Fremdeinschätzung durch betreuende Versorger. Allerdings gilt die Selbsteinschätzung durch den Patienten insbesondere zur Bewertung von subjektiven Symptomen wie Atemnot als Goldstandard [17, 23]. Dennoch konnte anhand von HOPE-Daten bereits zumindest über das Vorhandensein von Atemnot eine gute Übereinstimmung zwischen Patienten und Ärzten sowie Pflegekräften gezeigt werden [29].
Zuletzt stellt die hohe Anzahl an fehlenden Daten bezüglich der Abschlussinformationen (24,7\%) eine Einschränkung der sich darauf beziehenden Ergebnisse dar.

\section{Schlussfolgerung}

Jeder zweite Patient einer stationären spezialisierten Hospiz- und Palliativeinrichtung in Deutschland leidet unter Atemnot. Atemnot ist damit ein relevantes Symptom in der klinischen Palliativversorgung. Atemnot ist mit einem schlechteren Funktionsstatus, einer höheren Symptomlast, vermehrtem Hilfebedarf bei Aktivitäten des täglichen Lebens und einer geringeren Überlebenszeit betroffener Patienten assoziiert.

Der hohen klinischen Relevanz dieses belastenden Symptoms stehen eine immer noch verbesserungsfähige Versorgung und Behandlung gegenüber um die Lebensqualität von atemnötigen Patienten bestmöglich zu erhalten- hier bedarf es einer gemeinsamen und verstärkten Forschungsanstrengung, um wirksame Therapieverfahren zu entwickeln und in die klinische Versorgung zu integrieren.

\section{Konsequenzen für Klinik und Praxis}

- Jeder zweite Patient einer spezialisierten stationären Hospiz- oder Palliativeinrichtung leidet unter Atemnot.

- Patienten mit Atemnot hatten im Vergleich zu Patienten ohne Atemnot einen niedrigeren Funktionsstatus, litten unter einer höheren Symptomlast und hatten eine höhere Mortalität.

- Die klinische Versorgung von Patienten mit Atemnot bedarf einer höheren Aufmerksamkeit. Mehr Bemühungen in der klinischen Forschung sind nötig, um Atemnot effektiv symptomatisch lindern zu können.

\section{Danksagungen}

Die präsentierten Daten sind Teil einer Doktorarbeit von NA zur Erlangung des Doktorgrades der Medizinischen Fakultät der Georg-August-Universität zu Göttingen [4]. Die Ergebnisse wurden als Vortrag auf dem 6th EAPC research congress in Glasgow 2010 und als Poster auf dem 8 . Kongress der Deutschen Gesellschaft für Palliativmedizin „Grenzen überwinden“ in Dresden 2010 präsentiert. Für die statistische Beratung bedanken wir uns ausdrücklich bei Dr. Gao Wei (London) und Prof. Dr. Martin Helmich (Köln).

\section{Finanzierung}

Die Hospiz und Palliativerhebung (HOPE) wurde unterstützt von der Mundipharma gmbH Limburg. 


\section{Interessenskonflikte}

STS erhielt Honorare für einen CME Artikel über Atemnot vom Deutschen Ärzteblatt, eine Erstattung von Teilnahmegebühren für den 10 . Kongress der Deutschen Gesellschaft für Palliativmedizin (DGP) 2014 in Düsseldorf wegen Vorträgen über Atemnot und Atemnotattacken und ist Projektleiter der S3-Leitlinie Palliativmedizin (inklusive einem Kapitel zum Thema Atemnot).

STS und und RV erhielten Gelder für eine selbst initiierte klinische Studie (Investigator Initiated Trial) zur Behandlung von Atemnotattacken von TEVA $\mathrm{GmbH}$.

RV ist Beteiligter an der S3-Leitlinie Palliativmedizin. CB erhielt ein Honorar für einen CME Artikel über Atemnot vom Deutschen Ärzteblatt sowie für Vortrags-und Fortbildungsveranstaltungen vom Bürgerhospital Stuttgart, Reisekostenerstattungen von der Uniklinik Köln und vom Bürgerhospital Stuttgart, Gelder für ein selbstinitiiertes Forschungsprojekt vom Bundesministerium für Bildung und Forschung und ist Leiterin der AG Dyspnoe der S3-Leitlinie Palliativmedizin.

VW hat eine Reisekostenerstattung von TEVA für eine Kongressreise (8th World Research Congress of the EAPC, Lleida, Spanien, 05.-07.06. 2014) außerhalb der hier berichteten Arbeit erhalten.

GL erhielt Honorare für Beratungstätigkeiten von an HOPE teilnehmenden Institutionen, hat Teilnahmegebühren für einen Kongress und Honorar für Vorträge im Bezug zum Thema HOPE von der DGP erstattet bekommen.

FN hat für die Firmen Archimedes und Mundipharma nicht produktbezogene Vorträge gehalten und Vortragshonorare erhalten und 2014 einen Vorsitz für die Firma TEVA beim Kongress der DGP übernommen, für den ein Honorar gezahlt wurde.

\section{Literatur}

1 Ahmed T, Steward JA, O'Mahony MS. Dyspnoea and mortality in older people in the community: a 10-year follow-up. Age Ageing 2012; 41: 545-549

2 Alt-Epping B, Pache S, Lindena G et al. Anticancer therapies in specialized palliative care - a multicenter survey. Support Care Cancer 2012; 20: 2385-2389

3 Alt-Epping B, Staritz AE, Simon ST et al. What is special about patients with lung cancer and pulmonary metastases in palliative care? Results from a nationwide survey. J Palliat Med 2012; 15: 971-977

4 Altfelder N. Charakteristika von Palliativpatienten mit Atemnot - Ergebnisse der Hospiz- und Palliativerhebungen (HOPE) von 2006 bis 2008 [Dissertation]. Göttingen: Georg-AugustUniversität zu Göttingen; 2012

5 Bajwah S, Higginson IJ, Ross JR et al. Specialist palliative care is more than drugs: a retrospective study of ILD patients. Lung 2012; 190: 215-220

6 Bausewein C, Booth S, Gysels M et al. Understanding breathlessness: cross-sectional comparison of symptom burden and palliative care needs in chronic obstructive pulmonary disease and cancer. J Palliat Med 2010; 13: 1109-1118

7 Cancer Research UK. Lung cancer survival statistics 2013.

http://www.cancerresearchuk.org/

health-professional/lung-cancer-survival-statistics Letzter Zugriff am 30.10.2014

8 Cohen J. Statistical Power Analysis for the Behavioral Sciences. 2. Aufl. Hillsdale: Lawrence Erlbaum Associates; 1988
9 Cuervo Pinna MA, Mota Vargas R, Redondo Moralo $\mathrm{M}$ ] et al. Dyspnea - a bad prognosis symptom at the end of life. Am J Hosp Palliat Care 2009; 26: 89-97

10 Currow DC, Smith J, Davidson PM et al. Do the trajectories of dyspnea differ in prevalence and intensity by diagnosis at the end of life? A consecutive cohort study. J Pain Symptom Manage 2010; 39: 680-690

11 Figarska SM, Boezen HM, Vonk JM. Dyspnea severity, changes in dyspnea status and mortality in the general population: the Vlagtwedde / Vlaardingen study. Eur Epidemiol 2012; 27: 867-876

12 Frostad A, Soyseth V, Haldorsen T et al. Respiratory symptoms and 30 year mortality from obstructive lung disease and pneumonia. Thorax 2006; 61: 951-956

13 Geldmacher H, Biller H, Herbst A et al. Die Prävalenz der chronisch obstruktiven Lungenerkrankung (COPD) in Deutschland. Dtsch Med Wochenschr 2008; 133: 2609-2614

14 Giacomini M, Dejean D, Simeonov D et al. Experiences of living and dying with COPD: a systematic review and synthesis of the qualitative empirical literature. Ont Health Technol Assess Ser 2012; 12: 1-47

15 Gysels M, Bausewein C, Higginson IJ. Experiences of breathlessness: a systematic review of the qualitative literature. Palliat Support Care 2007; 5: 281-302

16 Hayes AW, Philip J, Spruyt OW. Patient reporting and doctor recognition of dyspnoea in a comprehensive cancer centre. Intern Med J 2006; 36: 381-384

17 Hui $D$, Morgado M, Vidal M et al. Dyspnea in Hospitalized Advanced Cancer Patients: Subjective and Physiologic Correlates. J Palliat Med 2013; 13: 274-270

18 lyer S, Roughley A, Rider A et al. The symptom burden of non-small cell lung cancer in the USA: a real-world cross-sectional study. Support Care Cancer 2014; 22: 181-187

19 Iyer S, Taylor-Stokes G, Roughley A. Symptom burden and quality of life in advanced non-small cell lung cancer patients in France and Germany. Lung Cancer 2013; 81: 288-293

20 Moens K, Higginson IJ, Harding R. Are There Differences in the Prevalence of Palliative Care-Related Problems in People Living With Advanced Cancer and Eight Non-Cancer Conditions? A Systematic Review. J Pain Symptom Manage 2014; 48: 660-677

21 Nauck F, Ostgathe C, Klaschik E et al. Drugs in palliative care: results from a representative survey in Germany. Palliat Med 2004; 18: 100-107

22 Pallant J. SPSS Survival manual. 3. Aufl. Berkshire: Open University Press; 2007

23 Parshall MB, Schwartzstein RM, Adams L et al. An Official American Thoracic Society Statement: Update on the Mechanisms, Assessment, and Management of Dyspnea. Am J Respir Crit Care Med 2012; 185: 435-452

24 Radbruch L, Sabatowski R, Loick G et al. MIDOS Validierung eines minimalen Dokumentationssystems für die Palliativmedizin. Schmerz 2000; 14 : 231-239

25 Reddy SK, Parsons HA, Elsayem A et al. Characteristics and correlates of dyspnea in patients with advanced cancer. J Palliat Med 2009; 12: 29-36

26 Reuben DB, Mor V. Dyspnea in terminally ill cancer patients. Chest 1986; 89: 234-236

27 Robert Koch Institut, Gesellschaft der epidemiologischen Krebsregister in Deutschland e. V. Krebs in Deutschland 2009/2010. 9 Ausgabe, 2013 
28 Shin JA, Kosiba JD, Traeger L et al. Dyspnea and Panic Among Patients With Newly Diagnosed Non-Small-Cell Lung Cancer. J Pain Symptom Manage 2014; 48: 465-470

29 Simon ST, Altfelder N, Alt-Epping B et al. Is breathlessness what the professional says it is? Analysis of patient and professionals' assessments from a German nationwide register. Support Care Cancer 2014; 22: 1825-1832

30 Smith EL, Hann DM, Ahles TA et al. Dyspnea, anxiety, body consciousness, and quality of life in patients with lung cancer. I Pain Symptom Manage 2001; 21: 323-329

31 Statistisches Bundesamt. Bevölkerung nach Altersgruppen Deutschland, 2014 https://www.destatis.de/DE/ZahlenFakten/ GesellschaftStaat/Bevoelkerung/ Bevoelkerungsstand/Tabellen_/lrbev01.html Letzter Zugriff am 09.03.2016

32 Stiel S, Bertram L, Neuhaus S et al. Evaluation and comparison of two prognostic scores and the physicians' estimate of survival in terminally ill patients. Support Care Cancer 2010; 18: 43-49
33 Stiel S, Pollok A, Elsner $F$ et al. Validation of the Symptom and Problem Checklist of the German Hospice and Palliative Care Evaluation (HOPE). J Pain Symptom Manage 2012; 43: 593-605

34 Trajkovic-Vidakovic M, de Graeff A, Voest EE et al. Symptoms tell it all: a systematic review of the value of symptom assessment to predict survival in advanced cancer patients. Crit Rev Oncol Hematol 2012; 84: 130-148

35 Walsh D, Donnelly S, Rybicki L. The symptoms of advanced cancer: relationship to age, gender, and performance status in 1,000 patients. Support Care Cancer 2000; 8: 175-179

36 Zimmermann C, Burman D, Follwell M et al. Predictors of symptom severity and response in patients with metastatic cancer. Am J Hosp Palliat Care 2010; 27: 175-181
DOI 10.1055/s-0041-106678 Dtsch Med Wochenschr 2016; 141: e87-e95 (c) Georg Thieme Verlag KG . Stuttgart · New York . ISSN 0012-0472 